\title{
Investigation of effect of oblique ridges on heat transfer in plate heat exchangers
}

\author{
Jan Novosád ${ }^{1, a}$ and Václav Dvořák ${ }^{1}$ \\ ${ }^{1}$ Department of Power Engineering Equipment, Faculty of Mechanical Engineering, Technical University of Liberec, \\ Studentska 2, 46117, Liberec, Czech Republic
}

\begin{abstract}
This article deals with numerical investigation of flow in plate heat exchangers. These are counterflow heat exchangers formed by plates. These plates are shaped by the ridges to intensify heat transfer. The objective of the work is the investigation of effect of straight oblique triangular ridges for increasing of heat transfer and pressure losses. The ridges on adjacent plates intersect and thus form a channel of complex shape. The research includes various types of ridges with different fillets and ridges spacing. The work also investigates the number of ridges that is necessary for optimization calculations. Obtained data are analysed and the heat transfer coefficient and pressure loss are evaluated. Conclusion describes the effect of fillets, ridges pitch and number of ridges.
\end{abstract}

\section{Introduction}

The development of recuperative heat exchangers in recent years focused on increasing efficiency. Another challenge is the development of so-called enthalpy exchangers for simultaneous heat and moisture transport, i.e. transport of both sensible and latent heat. An analysis of potential energy savings for conditioning fresh air with a membrane-based energy recovery ventilator was presented by Nia and Zhang in works [1] and [2]. They calculated the recovered sensible and latent energy and compared it with the energy required to condition fresh air. The result was that in hot and humid regions latent energy recovery dominates the total energy recovery effects. High sensible efficiency of a heat exchanger is insignificant unless the latent heat is recovered at the same time.

The development of heat exchanger includes also investigation of suitable methods for testing of materials. Dvorak and Novotny in work [3] developed a method for determination of very low permeability of membranes to air. The used a novel device to test very low values of permeability based on measuring of pressure drop in a pressurised vessel and derived that the pressure drop in the system in time is exponential and the exponent is proportional to the value of permeability. In work [4], Novotny et al. presented a method and a device for testing of moisture transport, which can simulate forced counter-flow in real heat exchangers. From experimentally obtained data Vestfalova in work [5] derived material properties determining the moisture transfer. According these results, the material for a novel membrane based heat exchanger was chosen.

Now it is necessary to optimize the shape of plates of the heat exchanger. The heat transfer surface of plate is not usually smooth, but is provided with stumps and ridges to intensify the heat transfer. The objective of optimization is not only to increase the transfer of heat or moisture but also to reduce pressure losses or material costs.

The aim of this work is to numerically investigate effects of straight but oblique triangular ridges on pressure losses and heat transfer. The ridges on adjacent plates intersect and thus form a channel of complex shape. The research includes various types of ridges with different fillets and ridges spacing. Later the heat exchanger plates will by optimized.

\section{Numerical simulation}

The subject of investigation is the air flow in channels that are formed between two plates. Every plate separates fresh air and waste air. The heat transfer from hotter airs to the colder one is realized through the mass of the plate.

This study simulates the operation of a heat recovery unit in counter flow heat exchanger during the winter season, so the corresponding parameters of simulation are chosen. Fresh air temperature is $0\left({ }^{\circ} \mathrm{C}\right)$, the temperature of waste air is $20\left({ }^{\circ} \mathrm{C}\right)$. Velocity in the input cross sectional area of the heat exchanger is chosen as $v=2.5\left(\mathrm{~m} \cdot \mathrm{s}^{-1}\right)$. Outflows are specified as barometric pressure.

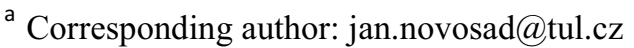




\subsection{Geometry of models and meshing}

Plates of the exchanger are shaped by triangular ridges, which are oblique to the direction of the main flow. Sketch of the shape is shown in figure 1. Modifications of plates are realised by changing of counts of ridges, the ridges pitch and theirs fillet. Other dimensions are the same for all cases, see figure 1.

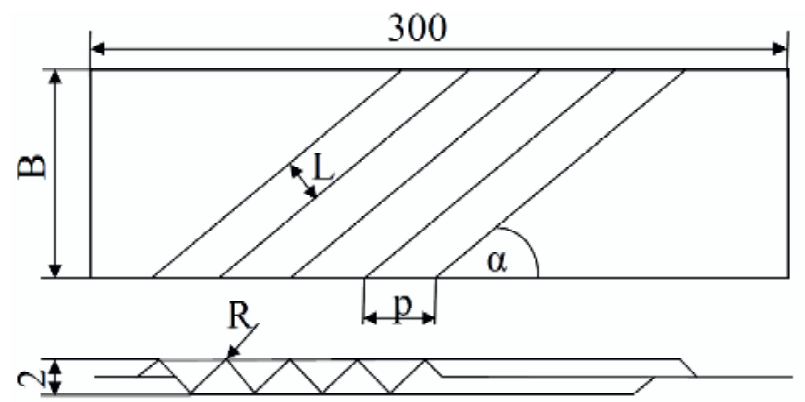

Figure 1.Basic geometry of the plate.

The research analyses the effect of the fillet size of the ridges $R \quad(\mathrm{~m})$, which varies in interval of $R=\{0,0.5,1,2\}(\mathrm{mm})$. Zero value means sharp ridges with no fillet. Parameter $L(\mathrm{~m})$ is the distance between two ridges. It varies in interval of $L=\{4,6,8\}(\mathrm{mm})$. To simplify creation of the model geometry, the ridges pitch $p(\mathrm{~m})$ was defined. It is given by the equation

$$
p=\frac{L}{\cos 45^{\circ}}
$$

The size of the model width $B(\mathrm{~m})$ is prescribed by equation

$$
B=\frac{4 \cdot p}{\tan 45^{\circ}}
$$

with respect to other dimensions. Respecting this rule shall ensure crossing of the ridges at the edge of the model. The parameter values for each variant are shown in Table 1.

Table 1.Parameters of model geometry

\begin{tabular}{|c|c|c|}
\hline $\mathrm{L}(\mathrm{mm})$ & $\mathrm{p}(\mathrm{mm})$ & $\mathrm{B}(\mathrm{mm})$ \\
\hline 4 & 5.657 & 22.63 \\
\hline 6 & 8.485 & 33.94 \\
\hline 8 & 11.314 & 45.25 \\
\hline
\end{tabular}

Preparation of models and meshes was realized in ANSYS Workbench environment. The model was created in Design Modeler according to the above dimensions. Meshes were generated in Meshing module. Automatic functions were used to create hexahedral and tetrahedral mesh. Number of elements was approximately the same for all variants, it was about 1.3 million elements.

\subsection{Solution}

Fluid waste air considered as ideal gas with constant specific heat capacity $c_{p}=1006\left(\mathrm{~J} \cdot \mathrm{kg}^{-1} \cdot \mathrm{K}^{-1}\right)$. The energy equation was set to on. Viscous model SST k- $\omega$ was used. We used steady flow and pressure based solver. Named boundary conditions of the model are in figure 2. Unlabelled side surfaces are set to symmetry.

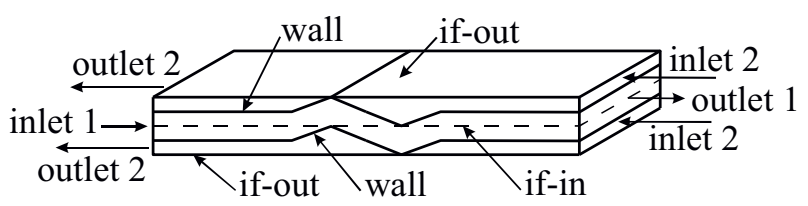

Figure 2.Boundary condition of the model.

Boundary condition types of named selection are shown in Table 2 . The value of the mass flow rate was set to represent the inlet velocity $\mathrm{v}=2.5\left(\mathrm{~m} \cdot \mathrm{s}^{-1}\right)$. The value is calculated from the equation of continuity

$$
\dot{m}=S \cdot \rho \cdot v
$$

where $S\left(\mathrm{~m}^{2}\right)$ is the area of inlet cross section and $\rho\left(\mathrm{kg} \cdot \mathrm{m}^{-3}\right)$ density of air.

After setting these boundary conditions, it is necessary to modify the mesh. It was saved to case file and then rotated by angle of $180^{\circ}$ around $\mathrm{x}$-axis. After that the original case file was appended. This process created model with crossed ridges. On this model we could set the interface and periodic interface boundary conditions.

Table 2.Parameters of boundary conditions

\begin{tabular}{|l|l|l|}
\hline Label & Boundary condition type & description \\
\hline inlet 1 & mass-flow-inlet & fresh air inlet \\
\hline inlet 2 & mass-flow-inlet & waste air inlet \\
\hline outlet 1 & pressure-outlet & fresh air outlet \\
\hline outlet 2 & pressure-outlet & waste air outlet \\
\hline if-in & interface & inner interface \\
\hline if-out & interface-periodic & outer interface \\
\hline symmetry & symmetry & symmetry surfaces \\
\hline wall & wall & plate \\
\hline
\end{tabular}

\subsection{Methodology of the results assessment}

The results obtained by numerical simulation were temperature and pressure fields. Values at inlet and outlet boundary conditions were evaluated using Mass-weighted-average method.

To evaluate values of quantities along the flow, cross sections of the channel with different $\mathrm{x}$-coordinate were created. These sections were parallel with the inlet plane. 


\subsubsection{Assessment of the efficiency}

Efficiency of heat transfer $\eta(\%)$ was expressed by

$$
\eta=\frac{t_{1}-t_{2}}{t_{I 1}-t_{E 1}} \cdot 100
$$

where the index $I$ is for waste air (indoor), index $E$ for fresh air (exterior). Index 1 is the inlet, index 2 is outlet.

\subsubsection{Assessment of the pressure loss}

Pressure loss $\Delta p(\mathrm{~Pa})$ was the pressures difference between the inlet and outlet. Given that the input and output section had the same surfaces, the pressure loss could be calculated with as a difference of static pressures according to relation

$$
\Delta p=p_{S 1}-p_{S 2}
$$

Local loss coefficient $\xi(-)$ is used for the pressure loss assessment. It is the difference of total pressures between the inlet and outlet divided by dynamic pressure at the inlet

$$
\xi=\frac{p_{01}-p_{02}}{p_{D 1}}
$$

\subsubsection{Coefficient of heat transfer}

The dependence between heat balance and efficiency is expressed by

$$
\dot{m} \cdot c_{p} \cdot \Delta t \cdot \eta=k \cdot S \cdot \Delta t \cdot(1-\eta)
$$

where $\dot{m}\left(\mathrm{~kg} \cdot \mathrm{s}^{-1}\right)$ is the mass flow rate, $c_{p}\left(\mathrm{~J} \cdot \mathrm{kg}^{-1} \cdot \mathrm{K}^{-1}\right)$ is specific heat, $\Delta t\left({ }^{\circ} \mathrm{C}\right)$ is the temperature difference, $\eta$ is heat transfer efficiency, $k\left(\mathrm{~W} \cdot \mathrm{m}^{-2} \cdot \mathrm{K}^{-1}\right)$ heat transfer coefficient and $S\left(\mathrm{~m}^{2}\right)$ is the area of heat transfer surface. Equation (4) is substituted into equation (7). The result is relation between parameter $k \cdot S\left(\mathrm{~W} \cdot \mathrm{K}^{-1}\right)$ and efficiency expressed as

$$
k \cdot S=\dot{\mathrm{m}} \cdot \mathrm{c}_{\mathrm{p}} \cdot \frac{\eta}{1-\eta}
$$

Parameter $(k \cdot S)$ represents the efficiency of heat transfer. For showing the effect of count of ridges, all values are presented in the way

$$
\Delta Q_{N}=\frac{Q_{N}-Q_{N-n}}{n} .
$$

where $Q$ is evaluated quantity, $N$ is the count of ridges and $n$ is step (1, 2 or 4 ridges).

\section{Results}

\subsection{Effect of ridges count}

Static pressure distributions along plates are curried out in figure 3, where curves represent decrease of static pressure for different count of ridges $\mathrm{n}=\{0,1,4,8,12,16\}$. The curves show that the pressure loss increases for higher count of ridges. Every ridge causes additional local loss, while the friction loss remains almost the same.

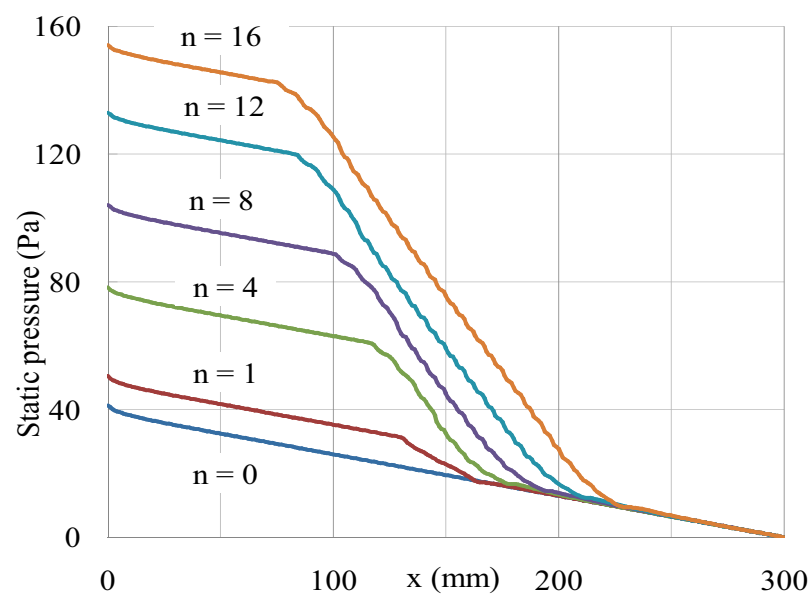

Figure 3. Static pressure distribution along plates for different number of ridges.

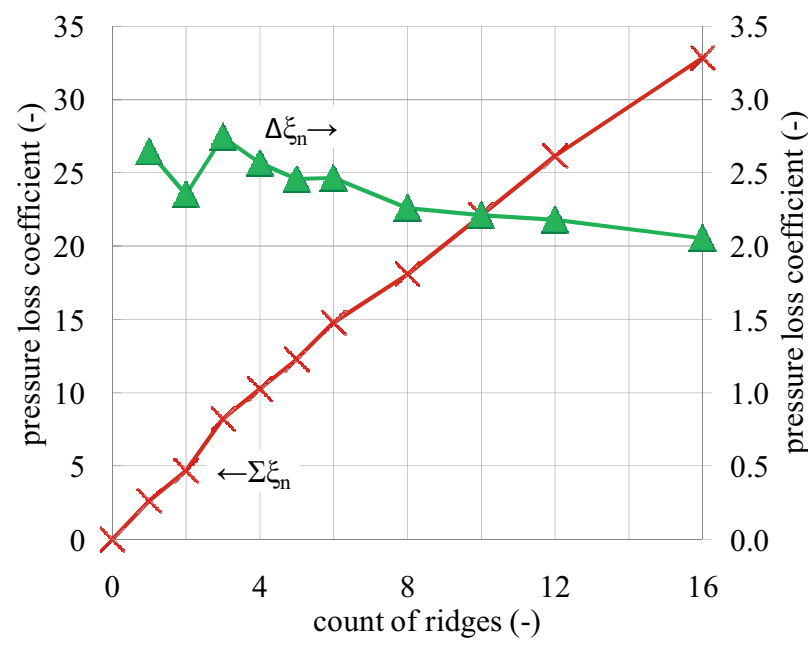

Figure 4. The dependence of local loss coefficient on count of ridges.

The first curve in figure 4 represents the total sum of local loss coefficient of all ridges. Of course, higher count of ridges causes higher pressure losses. The second curve represents pressure loss of each additional ridge. We can see that almost every next ridge causes lower pressure loss than the previous one, but the converged value was not reached. It seems that a higher count of ridges would be necessary. We can observe that the local loss coefficient oscillates for low count of ridges. Obviously, the count of ridges is too low 
in these cases and it is not sufficient enough for further result evaluation.

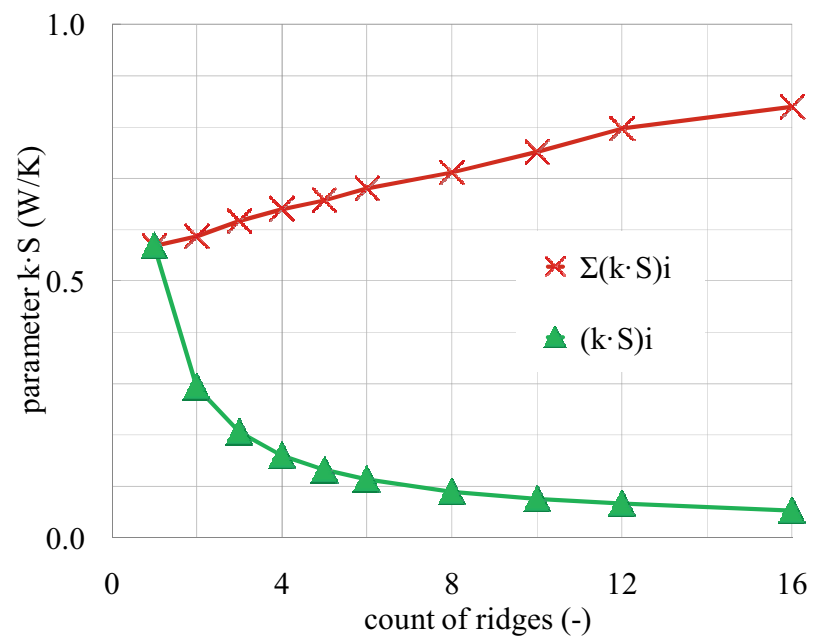

Figure 5. The dependence of parameter $(\mathrm{k} \cdot \mathrm{S})$ on number of ridges.

The dependence of parameter $(k \cdot S)$ on number of ridges is shown in figure 5. The first curve shows the total effect of all ridges. If the number of ridges is risen, the $(k \cdot S)$ parameter grows, so the heat transfer is more effective. The second curve shows effect of each additional ridge. We can see that each additional ridge has caused lower increase.

Figures 4 and 5 indicate some convergence to a value. We suppose, it is possible to find optimal number of ridges for which the effect of an additional ridge on pressure loss and parameter $(k \cdot S)$ will remain constant. This count of ridges would be appropriate for further optimization of the shape of heat transfer surfaces.

\subsection{Effect of fillet}

The effect of fillet on local loss coefficient is shown in figure 6. For higher fillets the local loss coefficient increases, but the value of increment decreases.

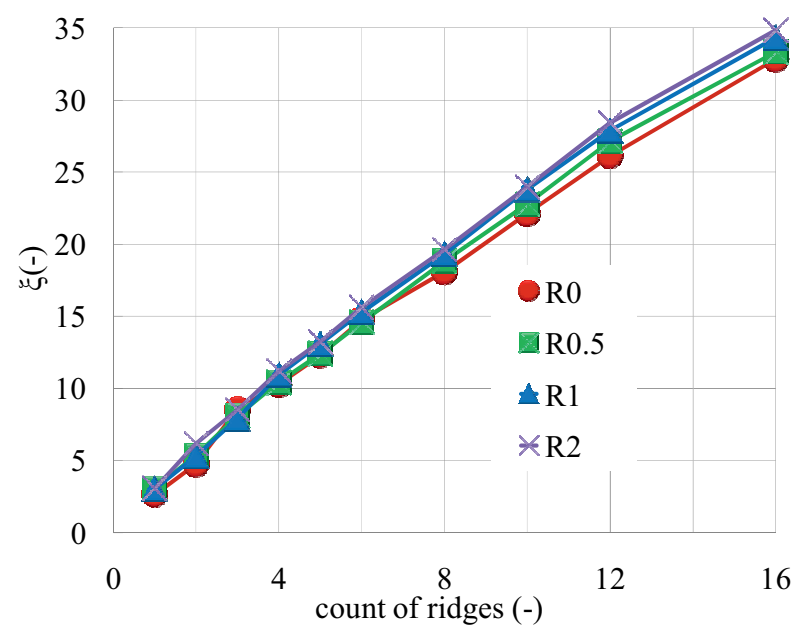

Figure 6. The dependence of total local loss coefficient on count of ridges for different fillet of the ridges.
Effect of fillet of ridges on parameter $(k \cdot S)$ is shown in figure 7. For number of ridges lower than eight, it is not possible to identify the best variant. This may be due to incomplete overlap of ridges in the peripheral parts of ridges. For number of ridges higher than eight, it can be said that the ridges with higher fillet is more efficient.

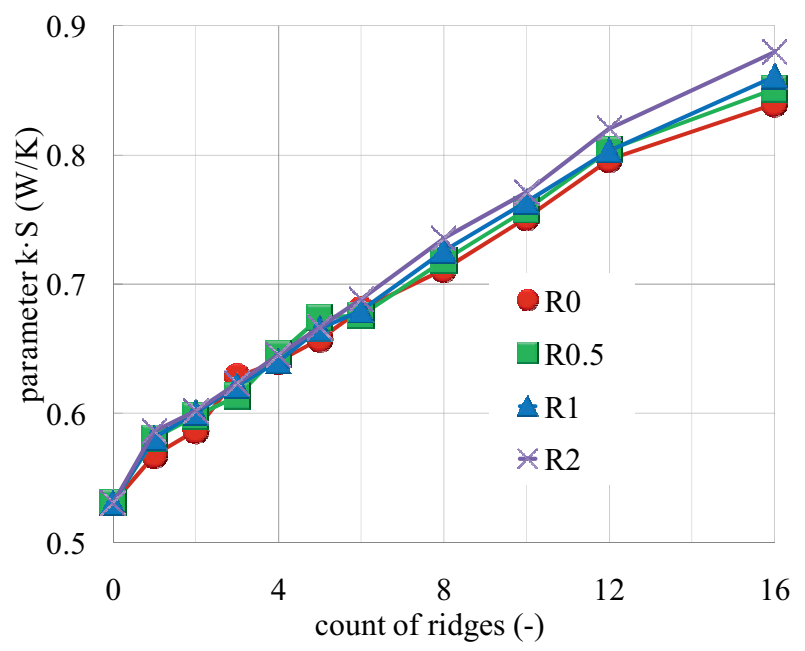

Figure 7. The dependence of parameter $(\mathrm{k} \cdot \mathrm{S})$ on count of ridges for different fillet of the ridges.

Figure 8 shows the dependence between the observed values of static pressure loss of the whole model and parameter $(k \cdot S)$. It illustrates the problem of optimization, because there are at least two objective functions: The total pressure loss, which is wanted to be as low as possible, and the total efficiency of heat transfer, which is wanted to be as high as possible. In general, we could state, that if the higher efficiency is required, the pressure loss increases for all modifications.

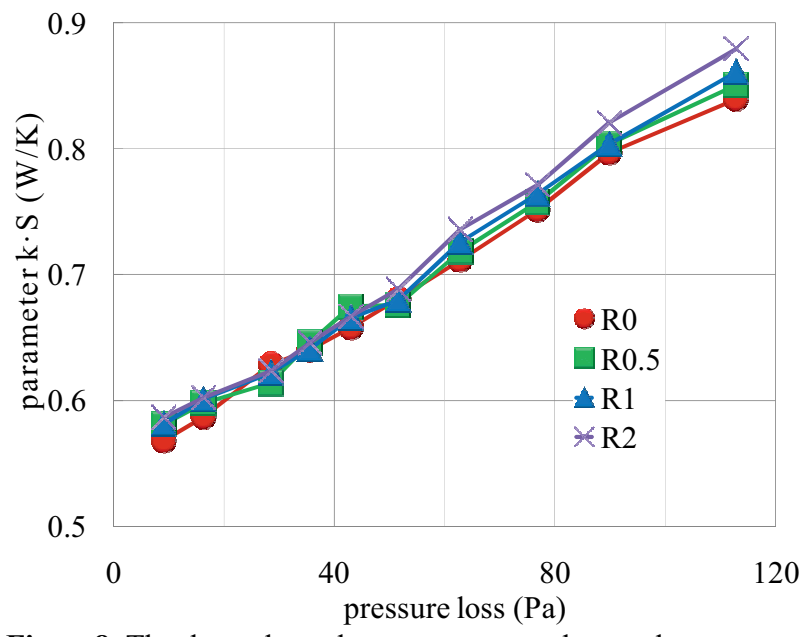

Figure8. The dependence between pressure loss and parameter $(\mathrm{k} \cdot \mathrm{S})$ for different count of ridges and different fillet of ridges.

\subsection{Effect of the ridges pitch}

To analyse the effect of the pitch, only cases with number of ridges to $n=\{8,10,12,16\}$ were investigated and simulated for the fillet $R=2(\mathrm{~mm})$. The dependence between pressure loss and parameter $(k \cdot S)$ is shown 
in figure 9. It shows that the pitch of $8(\mathrm{~mm})$ had better relation between pressure loss and efficiency than others modifications.

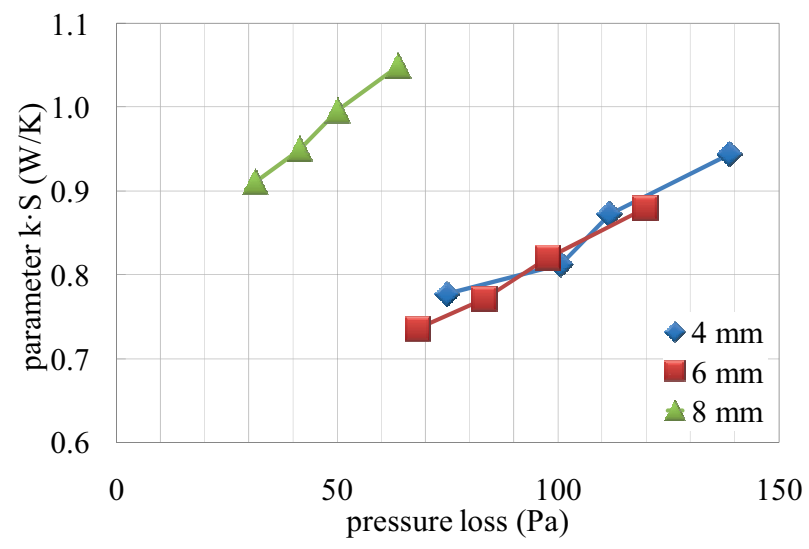

Figure 9.The effect of ridge pitch on parameter $(k \cdot S)$ and pressure loss for fillet $R=2(\mathrm{~mm})$ and count of ridges $n=\{8,10,12,16\}$.

\section{Conclusion}

Flow in plate heat exchanger was investigated numerically. Plates were shaped by ridges to intensify heat transfer. The effects of straight oblique triangular ridges for increasing of heat transfer and pressure losses were investigated. The influence of fillets, ridges spacing and count of ridges were researched. Obtained data were analysed and the heat transfer parameter $(k \cdot S)$ and pressure loss were evaluated.

It was found that the heat transfer and pressure losses significantly depend on number of ridges and that the effect of each additional ridge is lower than the effect of the previous one. It seems that the effects of additional ridge tend to certain values of pressure loss and parameter $(k \cdot S)$. To specify this, further investigation with more ridges will be performed on longer model.

Comparison of results with various rounding of ridges showed that bigger rounding yield higher efficiency of heat transfer and higher pressure losses. However, it seems that bigger fillets are preferential. Even bigger rounding or wavy line will be investigated in the next work.

Additional computations will be necessary to understand dependence of pressure loss and heat transfer on the ridge pitch. It seems that larger pits are better, but obviously an optimum must exists.

It is very difficult to gain optimal geometry, because we have two objective functions, the pressure loss and the efficiency of heat transfer. But it is obvious that if a heat exchanger with higher efficiency is required, it is necessary to use greater number of ridges, bigger rounding of edges and larger pitch. Generally, these arrangements will cause higher pressure losses. It is crucial to specify a unique objective function before an optimization procedure is started.

It would be beneficial to study the effect of the ridge angle, different value of pitch and different geometry of ridges, for example sinus function and half-circles, and its effect on heat exchanger parameters.

\section{Acknowledgement}

Author gratefully acknowledges financial support by Czech Technological Agency under the project TACR TA01020313 and project SGS 28000.

\section{References}

1. J.L. Niu, L.Z. Zhang, ASHRAE Trans. 108 (1) (2002)

2. L.Z. Zhang, J.L. Niu, Energy 26 (2001)

3. V. Dvorak, P. Novotny, SKMTaT 2013, University of Žilina, Slovak Republic.

4. P. Novotny, V. Nguyen, V. Dvořák, EFM12 Experimental Fluid Mechanics 2012, EPJ Web of Conferences, Volume 45

5. M. Vestfalova, EFM12 - Experimental Fluid Mechanics 2012, EPJ Web of Conferences, Volume 45 